\title{
DWDM-PON/mm-wave Wireless Converged Next Generation Access Topology using Coherent Heterodyne Detection
}

\author{
M.P. Thakur ${ }^{(1)}$, S. Mikroulis ${ }^{(1)}$, C.C. Renaud $^{(1)}$, J.E. Mitchell ${ }^{(1)}$, A. Stöhr ${ }^{(2)}$ \\ (1) University College London, Department of Electronic \& Electrical Engineering, WC1E 7JE, Torrington \\ place, London, United Kingdom \\ (2) Universität Duisburg-Essen, ZHO / Optoelectronics, Lotharstr. 55, 47057 Duisburg, Germany
}

\begin{abstract}
A radio-over-fibre system using coherent optical heterodyne detection scheme is proposed, to achieve seamless integration of a photonic Remote Antenna Unit (RAU) into a Next Generation Dense Wavelength Division Multiplexed Passive Optical Network (NG DWDM-PON). The proposed scheme significantly simplifies the optical mm-wave generation and data recovery as it doesn't require any high-bandwidth modulator at the central office or high-frequency Local Oscillators (LOs) at either the central office or the customer unit; or optical phase-locking techniques to generate the mm-wave wireless signal. A proof-of-concept transmission utilizing $1 \mathrm{~Gb} / \mathrm{s}$ On-Off Keying is experimentally demonstrated.
\end{abstract}

Keywords: radio over fiber, mm wave communications, coherent receiver, passive optical network

\section{INTRODUCTION}

With developments in broadband wireless networks, data rates up to $1 \mathrm{Gbit} / \mathrm{s}$ are expected to be supported over next generation access networks. Ever increasing wireless data rate has to be delivered from radio base stations whose reach scales inversely with the data rate. This situation will unavoidably lead to a higher density of base station deployment and a requirement for increased spectral efficiency, hence copper will no longer be suitable for the backhauling of the base station data. Fiber optical links based high bandwidth, cost-effective backhaul links will be required as a result to connect each of these base stations to a common central office (CO). Passive Optical Networks (PONs) can satisfy the requirements for increasingly investigated support of wireless backhauling [1]. However, longer-reach architectures, rather than the currently deployed legacy PONs, are anticipated to centrally connect base stations outside urban areas, leading to noticeable reduction in the number of expensive backhauling links. Recently FTTx standards have been produced to extend systems beyond time division multiplexing (TDM) into the wavelength division multiplexing (WDM) domain to provide increased capacity [2]. In addition to increased bandwidth, next-generation PONs also typically includes greater reach. NG-PON2 also explicitly proposed the inclusion of "optional wavelength division multiplexed (WDM) overlay extensions" to allow other services to be provided on a single optical distribution network.

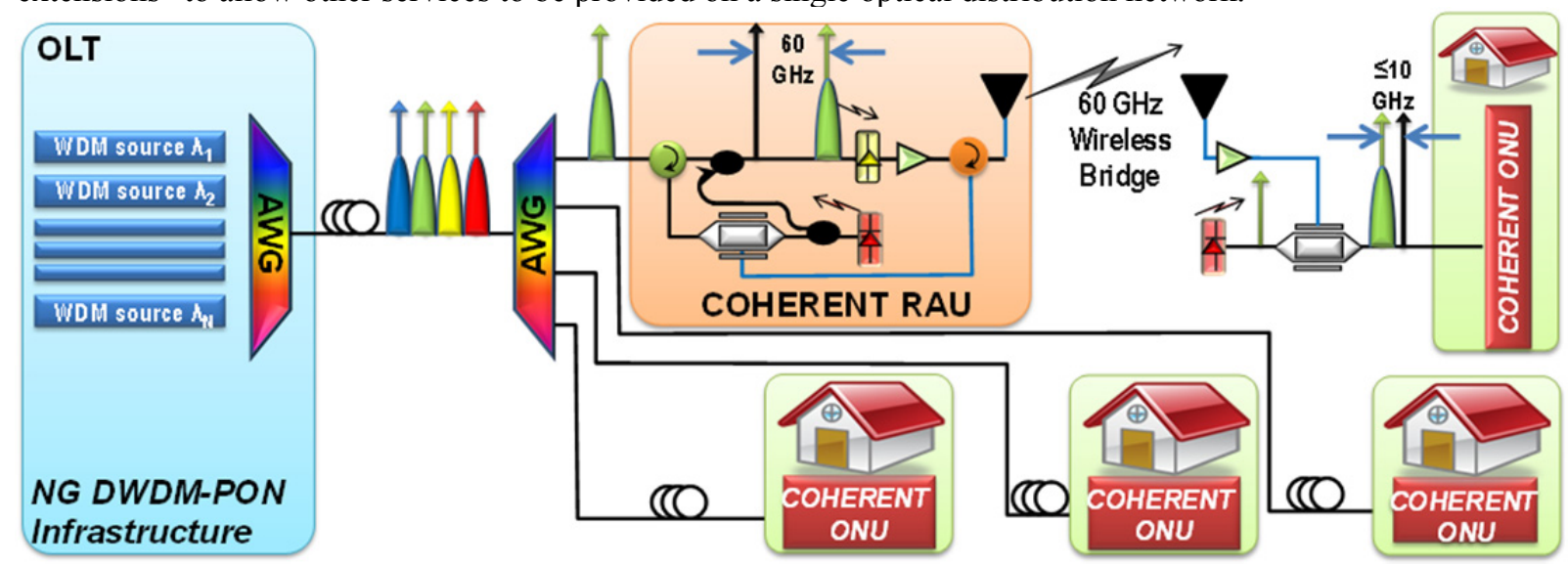

Figure 1. Next generation converged access topology using coherent detection DWDM scheme.

Further research is underway to develop a system that will support as many as 1000 wavelengths $(\sim 1 \mathrm{~Tb} / \mathrm{s}$ aggregate rate), each one, or more, dedicated to the user, in the $\mathrm{C}$ band using (ultra) dense wavelength division multiplexing UDWDM techniques [3]. This requires colorless coherent DWDM receivers to be developed. The additional gain provided by coherent reception means that systems with link budgets beyond $40 \mathrm{~dB}$ are possible. A second key advantage, is that the process of coherent reception inherently provides wavelength selectivity 
without the need for tunable filters at the receiver or Arrayed Waveguide Grating (AWGs) in the network, which are necessary in most WDM-PON implementations.

To support next generation wireless services, it is vital to integrate optical \& wireless technologies into a platform to combine the high fiber network capacity with the ubiquity and coverage of the wireless network. In recent years, radio-over-fiber (RoF) technology [4] has emerged as a promising candidate for bridging the gap between high mobility (wireless/mobile) and high capacity (optical) networks, offering the simplification of base stations (BSs) through the deployment of remote antenna units (RAUs), potentially reusing the already deployed FTTx for wireless access. Ongoing, a wireless bridge can be potentially used as a virtual fiber extension for places where fiber coverage is not possible.

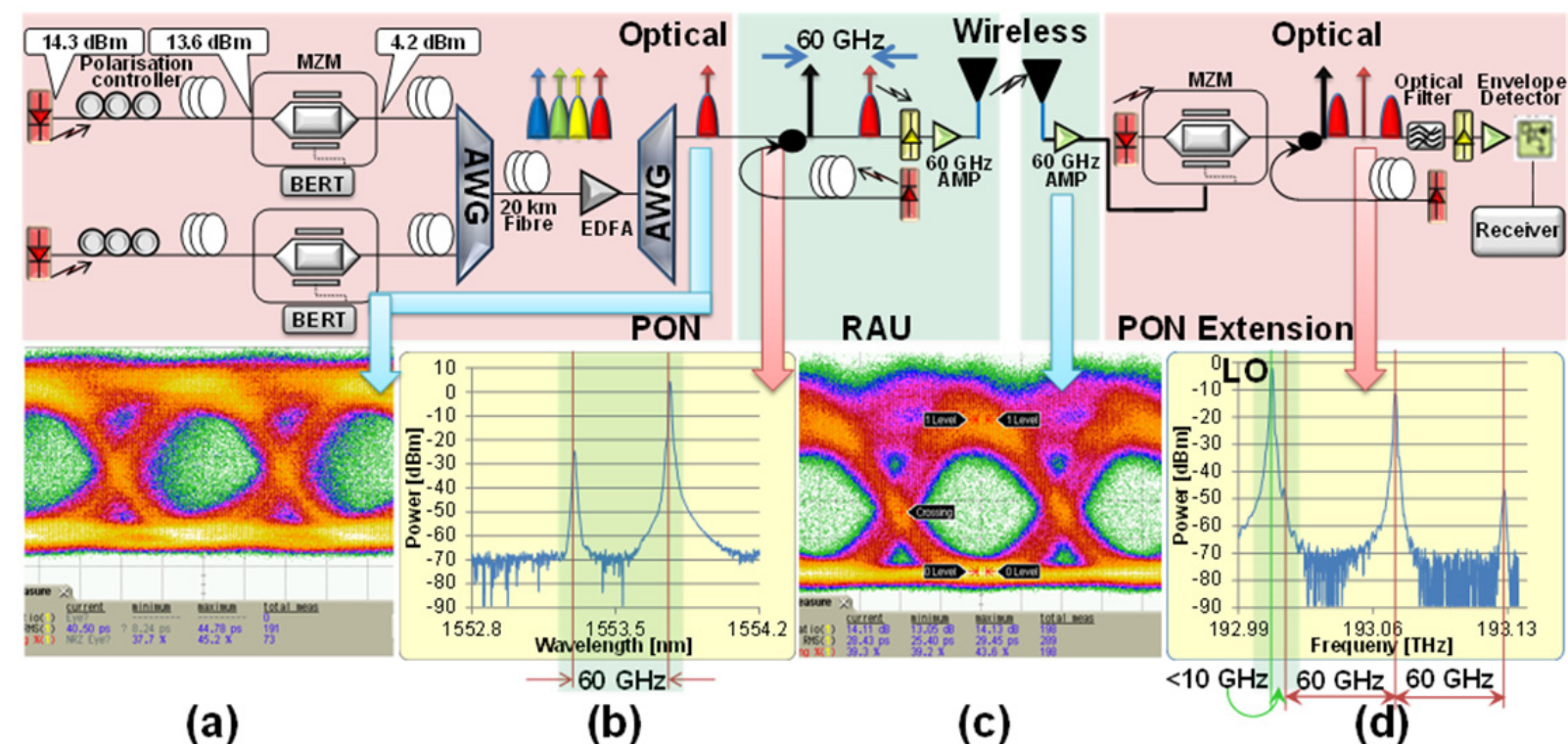

Figure2. Experimental system set up. (a)\& (b) eye diagram and optical spectrum after $20 \mathrm{~km}$ SSMF optical transmission and DEMUX, (c) eye diagram after O-E upconversion/coherent detection at the $70 \mathrm{GHz}$ photodiode and $60 \mathrm{GHz}$ wireless link propagation, (d) optical spectrum after E-O upconversion.

To leverage the ongoing development of UDWDM PON, a coherent optical heterodyne detection scheme is proposed (Fig. 1) and experimentally demonstrated in order to seamlessly integrate a photonic RAU into a coherent DWDM-PON scenario. Here, a multitude of users are connected to the core network either directly by a fiber connection or via a wireless connection fed by a fiber connection. Wireless access points could be femto base stations, pico cells or remotely connected radio heads ("Radio over fiber") providing broadband wireless access, A proof-of- concept experiment utilizing a $1 \mathrm{~Gb} / \mathrm{s}$ On-Off Keying (OOK) 60GHz wireless link embedded in a $50 \mathrm{GHz}$ grid DWDM-PON topology is performed, using single ordinary-linewidth thermally tuned lasers, potentially reused for the uplink transmission, and envelope detection techniques for the coherent receiver at the photonic RAU.

\section{EXPERIMENTAL SETUP}

The experimental system set up is depicted in Fig.2. A 6-channel externally modulated distributed feedback (DFB) laser scheme using an equal number of Mach-Zehnder Modulators (MZMs) is considered for the PON downlink. Each wavelength is modulated with $1 \mathrm{~Gb} / \mathrm{s}$ On-Off Keying (OOK) using a non-return to zero (NRZ) format. All WDM-PON channels are aggregated using an array waveguide grating (AWG) multiplexer (MUX) with a channel spacing of 50GHz, and subsequently amplified using an Erbium Doped Fiber Amplifier (EDFA), at the optical line terminal (OLT). A fiber link of $20 \mathrm{~km}$ of standard single mode fiber (SSMF) has been used between the OLT and the remote node (RN) where an AWG is used as a demultiplexer (DEMUX). A thermally tuned DFB laser is used as a local oscillator (LO) in an uncorrelated remote heterodyne detection (RHD) scheme [5], so as to optically generate the mm-wave using a $70 \mathrm{GHz}$ square-law photodiode. A wireless transmission of $5 \mathrm{~m}$ is performed. To allow characterization of the signal at this point, down-conversion of the mm-wave signal to baseband (BB) is performed by linearly detecting its envelope using a V-band waveguide broadband detector with a $3 \mathrm{GHz}$ electrical bandwidth.

To demonstrate the use of the signal as an optical link extension, the received optical signal, still on a $60 \mathrm{GHz}$ carrier is remodulated on to an optical carrier. This is performed using a $40 \mathrm{~Gb} / \mathrm{s} \mathrm{MZM}$ for post-transmission of the $\mathrm{mm}$ wave after the wireless bridge. Although, only specified for $40 \mathrm{~Gb} / \mathrm{s}$ operation, the frequency response of 
the device used extends to beyond $60 \mathrm{GHz}$ with an additional optical penalty of approximately $3 \mathrm{~dB}$. The signal is then carried over a short fiber link to an optical network unit (ONU) intended to represent a (U)DWDM customer device using coherent heterodyne detection. Here, a thermally tuned DFB laser is used as a local oscillator (LO) so as to coherently down-convert the signal from mm-wave to an IF and detect the signal on a low bandwidth photodetector. After optical filtering, down-conversion from IF to baseband and post processing is performed by using a digital envelope detector technique using a Tektronix Real time oscilloscope.

\section{RESULTS AND DISCUSSION}

The optical system performance is tested at various stages (Fig. 2) of the converged fiber-wireless coherent link, $(\mathrm{a}, \mathrm{b})$ after 20km SSMF optical transmission and DEMUX, (c) after O-E upconversion/coherent detection at the $70 \mathrm{GHz}$ photodiode and $60 \mathrm{GHz}$ wireless link propagation, and (d) after $60 \mathrm{GHz}$ wireless link/ E-O conversion and post optical transmission. The optical spectrum showing the optical carriers $60 \mathrm{GHz}$ apart and the eye diagram are depicted in Fig. 2 (a, b), while the eye diagram after wireless propagation can be seen in Fig 2 (c) showing an acceptable performance ( $\mathrm{Q}$ factor $>6$ ). The optical spectrum after mm-wave signal E-O conversion using a $40 \mathrm{~Gb} / \mathrm{s}$ MZM is shown in Fig. 2 (d), where both the optical carrier including $60 \mathrm{GHz}$ modulated side bands and the LO component appears, while in Fig. 3 (a) optical filtering is applied. The resulting eye diagram at this last stage is depicted in Fig. 3(b), where it is observed that the $1 \mathrm{~Gb} / \mathrm{s}$ signal is successfully detected after transmission at the composite optical-wireless-optical link.
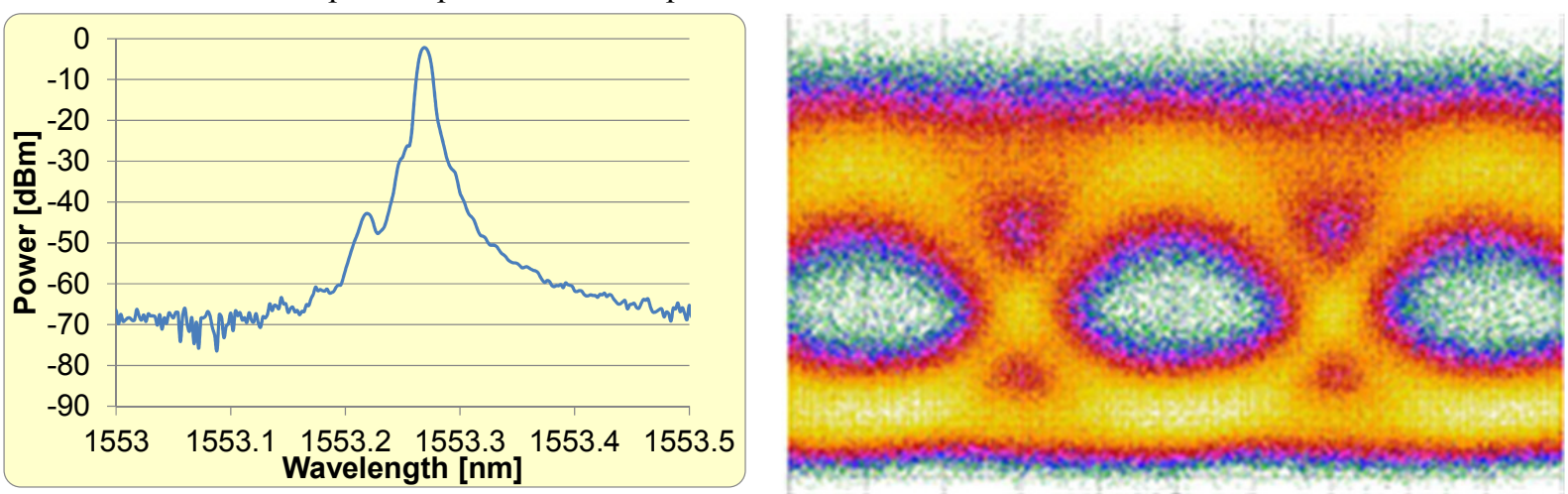

Figur3. Experimental results, (a) E-O conversion and optical filtering (b) eye diagram after 60GHz wireless link/ E-O conversion and post optical transmission).

\section{CONCLUSIONS}

An optical-wireless-optical link operating at $1 \mathrm{~Gb} / \mathrm{s}$ OOK based on a coherent optical heterodyne detection scheme has been proposed and experimentally demonstrated. The proposed system client side operates with a combination of transparent and low-cost alternatives i.e. low frequency detector after wireless bridge, instead of high frequency mixer and carrier-recovery and finally wavelength shift tolerance within the envelope detector frequency range. The wireless bridge provides a virtual fiber extension to a coherent NG DWDM PON deployment. The successful transmission shows that the concept is a promising approach towards heterogeneous fiber-wireless NG UDWDM PONs using colorless receivers.

\section{ACKNOWLEDGEMENT}

This research has been supported by European Union Seventh Framework project IPHOBAC-NG (619870) and the Marie Curie Fellowship FIRMWARE (327494).

\section{REFERENCES}

[1] M. Milosavljevic, M.P. Thakur, P. Kourtessis, J.E. Mitchell and J.M. Senior, "Demonstration of Wireless Backhauling Over Long-Reach PONs" J. Lightw. Technol. vol 30, no.5, pp.811,817, 2012.

[2] Y. Luo, X. Zhou, F. Effenberger, X. Yan, G. Peng, Y. Qian, and Y. Ma, "Time- and Wavelength-Division Multiplexed Passive Optical Network (TWDM-PON) for Next-Generation PON Stage 2 (NG-PON2)" J. Lightw. Technol. vol 31, no 4, pp. 587-593, 2013.

[3] D. Lavery, M Ionescu, S. Makovejs, E. Torrengo, S.J. Savory, “A long-reach ultra-dense 10 Gbit/s WDMPON using a digital coherent receiver" Optics Express, vol 18, no 25, pp. 25855-25860, 2010

[4] M. Parker, S. D. Walker, R. Llorente, M. Morant, M. Beltrán, I. Möllers, D. Jäger, C. Vázquez, D. Montero, I. Librán, S. Mikroulis, S. Karabetsos, A. Bogris, "Radio-over fibre technologies arising from the Building the future Optical Network in Europe (BONE) project" (2010)., IET Optoelectronics, vol. 4, No. 6, pp. 247-259, 2010.

[5] O. Omomukuyo, M. P. Thakur, and J. E. Mitchell, "Simple 60-GHz MB-OFDM ultrawideband RoF system based on remote heterodyning”, IEEE Photon. Technol. Let., Vol. 25, No. 3, pp. 268-271, 2013. 